\title{
Preservation of the DO W mass measurement to incorporate future PDF and physics models
}

\author{
Jakub Cúth* \\ (on behalf of $\mathrm{DO}$ collaboration) \\ Johannes Gutenberg Universität mainz \\ E-mail: cuth@uni-mainz.de \\ Matthias Schott \\ Johannes Gutenberg Universität Mainz \\ E-mail: schotteuni-mainz.de
}

The D0 experiment at the Fermilab Tevatron Collider provided in recent years one of the most accurate measurements of the $W$ boson mass. The precise knowledge of the $W$ boson mass, together with the mass of the Higgs Boson and the top quark, provides one of the most crucial tests of the Standard Model of particle physics. The uncertainties of this measurement are currently dominated by the limited knowledge of the parton density functions, which will improve in future years. Therefore, a dedicated effort is being made by the D0 Collaboration to preserve the $W$ boson mass analysis for a future reevaluation with improved proton descriptions and other improvements in the modeling of $W$ boson production and decay. We give an overview of this effort and discuss the underlying technical infrastructure. In addition, we also present a reevaluation of the $W$ boson mass measurement based on $\int L d t=4.3 \mathrm{fb}^{-1}$ of data with an updated PDF set as an example for future applications.

The European Physical Society Conference on High Energy Physics

22-29 July 2015

Vienna, Austria

*Speaker. 


\section{Introduction}

The measurement of the $W$ boson mass $\left(M_{W}\right)$ will be one of the most lasting scientific results of the D0 experiment, and it is expected to have an impact on the world average for at least the next decades. Even though the CERN Large Hadron Collider (LHC) experiments are currently in the process of preparing a new round of measurements, the expected experimental, theoretical, and model uncertainties will be, in the most optimistic scenario, on the order of $10 \mathrm{MeV}$. Hence the most recent measurements of $M_{W}$ at the D0 experiment [1] based on $\int L d t=4.3 \mathrm{fb}^{-1}$ of data with a value of $M_{W}=80.375 \pm 0.023 \mathrm{GeV}$ will be relevant even in the long term. It should be noted that the model uncertainty is $13 \mathrm{MeV}$, where $11 \mathrm{MeV}$ are due to the limited knowledge of parton density functions (PDFs). In fact, the measurement currently under preparation, which will use the full data set, will be dominated by these PDF uncertainties.

In addition, measurements at the Tevatron and the LHC are complementary to a large extent, as different eigenvectors of the underlying PDFs dominate the $W$ boson production in $p p$ and $p \bar{p}$ collisions. It is expected that the knowledge of PDFs will improve significantly in the future, as new measurements of the differential production cross-sections of $Z$ and $W$ bosons become available and will be used for future PDF fits. Therefore, it was the aim of this work, which is summarized in the following, to preserve the D0 $W$ boson mass analysis, allowing a reevaluation of the $M_{W}$ measurement using newer PDF sets and a reduction of the overall systematic uncertainty.

This paper is structured as follows. In Section 2, we introduce the $W$ mass measurement methodology and in Section 3, we describe the technical infrastructure which was purchased and installed at the University of Mainz for the preservation of the $W$ boson mass analysis. Also, the software details are briefly described, followed by an example of the usage of the preserved system for the reevaluation of PDF uncertainties with different PDF sets.

\section{Measurement of $W$ mass with D0 at the Tevatron collider}

The Fermilab Tevatron Collider, the most powerful $p \bar{p}$ collider with $\sqrt{s}=1.96 \mathrm{TeV}$, delivered its last collisions in September 2011. In this document, we are focusing on the data taking period from the summer of 2006 to the summer of 2009, in which the D0 detector [2] collected data corresponding to $\int L d t=4.3 \mathrm{fb}^{-1}$. The D0 detector has an inner tracking system, a calorimeter, and a muon system. The measurement of the $W$ boson mass is exclusively performed in the electron decay channel. The value is extracted via a template fit approach, using kinematic distributions of the decay products: the electron transverse momentum $p_{T}(e)$ and the transverse mass $m_{T}$. The $m_{T}$ is defined by

$$
m_{T}=\sqrt{2 p_{T}(e) \mathscr{E}_{\mathrm{T}}(1-\cos \Delta \varphi)},
$$

where $\Delta \varphi$ is the angle between the electron and the missing energy $\mathbb{E}_{\mathrm{T}}$ in the transverse plane. The $W$ and $Z$ boson production and decay kinematics are simulated using the RESBOS generator [3] with the CTEQ6.6 [6] where the final state photon radiation is included via PHOTOS [4]. The detector response is simulated by a parametrized Monte Carlo simulation (FastMC), which has been specifically developed for this analysis and tuned by the $Z \rightarrow e e$ data control sample and the full Monte Carlo simulation of the detector. 
Since the modeling of the detector response will not change in the future, it is not necessary to reestimate the detector-related uncertainties of $M_{W}$. However, it may be advantageous to use new programs which allow for an accurate prediction of the vector boson production and decay kinematics. Therefore, our goal is to implement a solution which preserves the various functionalities of the code, but also allows for a reevaluation of theoretical model uncertainties via the incorporation of items such as $p_{T}(W)$ distributions or PDF sets.

\section{Preservation of the analysis}

The D0 collaboration is preparing long term storage of data and code for all major analyses. In addition to this centralized collaboration wide effort, it was decided to provide an independent method to rerun the $M_{W}$ analysis, since it uses a large extent of highly specific code developments, e.g. the FastMC. To achieve this goal, a dedicated server for the $M_{W}$ analysis preservation has been set up at the university of Mainz. The basic hardware parameters of the server are:

- CPU: 2x AMD Opteron 6344 (2,60 GHz, 12-core, $16 \mathrm{MB})$

- RAM: 4x Kingston DIMM 8 GB PC3-10600 ECC Reg.

- HDD: 4x 3000 GB HGST UltraStar 7K4000 Enterprise S-ATA (64 MB, 7.200, S-ATA 6Gb/s) LSI with MegaRAID 9266-4i SAS/S-ATA 6Gb/s Controller

- Server-Mainboard: with AMD SR5690/SP5100 Chipset (E-ATX), on board: VGA, 2x GbitLAN, 6x S-ATA 3Gb/s, RAID;

This server contains all relevant data and code. In order to allow for a working operating system environment in the upcoming years, a VirtualBox [5] implementation was used. The VirtualBox can execute the operating system used by the analysis and thus allow for the compilation and the execution of the full D0 analysis software. To make the interface user-friendly, we provide a basic set of scripts for the execution of the different analysis steps. Detailed documentation and help-pages are available directly after the login on the dedicated server. This also allows newcomers to rerun the D0 $W$ boson mass analyses. The reproducibility of the analyses has been extensively tested. As an example, the resulting kinematic distributions of $p_{T}(e)$ and $m_{T}$ after the $M_{W}$ fit using the preservation analysis and the stored data are showed in Figure 1. They are in perfect agreement with the published results [1].

As an example of the reevaluation of $M_{W}$ with a different PDF set, we determined $M_{W}$ using the MSTW08NLO PDF set [7] instead of CTEQ6.1 [8], which was used in the published analysis [1]. The results of $M_{W}$ are shown in Figure 2, where the published values of $M_{W}$, the preserved values and the reevaluated values are indicated. 

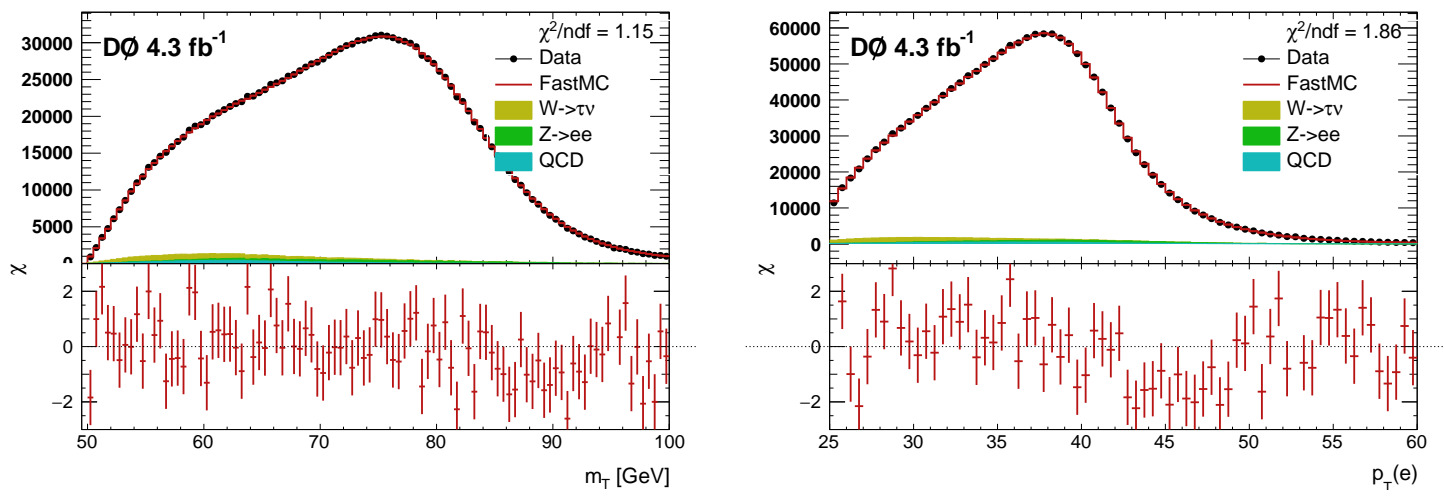

Figure 1: The distributions used for extracting $M_{W}$ obtained from the preserved code (FastMC) and data showing the reproducibility of the published results. The plotted distributions from left to right are: transverse mass of the $W$ and transverse momentum of the electron. In lower part of each plot the $\chi$ value between the data and FastMC is shown for each bin.

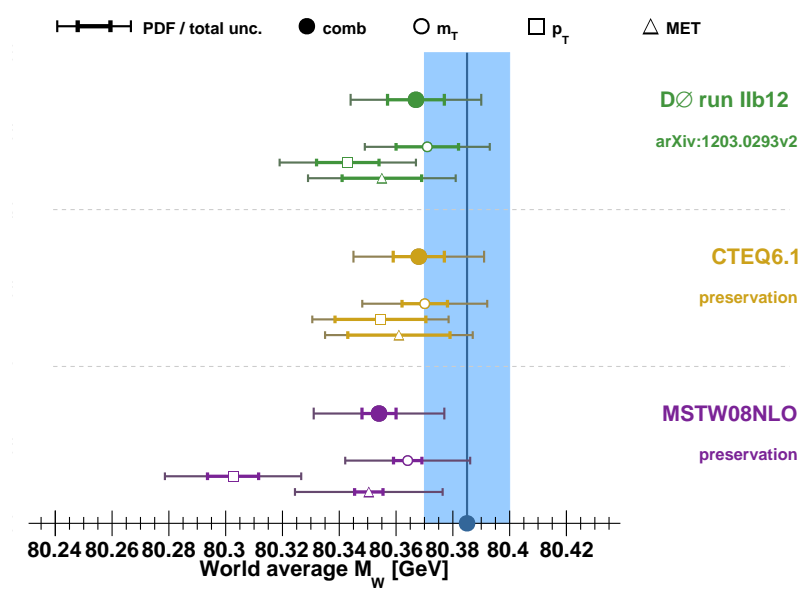

Figure 2: Fitted $W$ boson mass using different PDF sets. The published result (in green) is compared to results from preservation (yellow for CTEQ6.1 and purple for MSTW08NLO). The current world average [9] is represented by the blue band.

\section{Conclusion}

The preservation of the $W$ mass measurement at the D0 detector allows the option to reevaluate the $W$ boson mass using new parton density functions of the proton in future years. To provide the necessary infrastructure, a new server was installed at the University of Mainz in June 2014. It stores all data and software and is purely dedicated to the D0 $W$ mass analysis preservation. For data-safety reasons, a mirror of this setup is duplicated at the Mogon-computer cluster infrastructure at the university of Mainz, guranteeing a long term preservation of the analysis infrastructure. The setup has been extensively tested and has been demonstrated to successfully complete a reevaluation of the $W$ boson mass with a different PDF set, allowing for potential future reductions in the theoretical uncertainties of the $W$ boson mass value. 


\section{References}

[1] V.M. Abazov et al. [D0 collaboration], Phys. Rev. Lett. 108, 151804 (2012).

[2] V. M. Abazov et al. [D0 Collaboration], Nucl. Instrum. Methods Phys. Res., Sect. A 565, 463 (2006); M. Abolins et al., Nucl. Instrum. Methods Phys. Res., Sect. A 584, 75 (2008); R. Angstadt et al., Nucl. Instrum. Methods Phys. Res., Sect. A 622, 298 (2010).

[3] C. Balazs and C. P. Yuan, Phys. Rev. D 56, 5558 (1997).

[4] P. Golonka and Z. Was, Eur. Phys. J. C 45, 97 (2006).

[5] Oracle VM VirtualBox, https://www.virtualbox.org/

[6] P. M. Nadolsky, H. L. Lai, Q. H. Cao, J. Huston, J. Pumplin, D. Stump, W. K. Tung and C.-P. Yuan, Phys. Rev. D 78, 013004 (2008).

[7] A. D. Martin, W. J. Stirling, R. S. Thorne and G. Watt, Eur. Phys. J. C 63, 189 (2009).

[8] D. Stump et al. [CTEQ group], JHEP 0310, 046 (2003) .

[9] K.A. Olive et al. [Particle Data Group], Chin. Phys. C 38, 090001 (2014). 